\title{
Pre-cochlear Implantation Aural/Oral Rehabilitation, Is it Mandatory?
}

\author{
Rabeea Al Sabeela ${ }^{1}$, Safinaz Nagib Azab ${ }^{2 *}$ \\ ${ }^{1}$ Professor of Otorhinolaryngology, King Abd El-Aziz Specialized Hospital, Kingdome of Saudi Arabia \\ ${ }^{2}$ Professor of Phoniatrics, Department of Otorhinolaryngology, Faculty of Medicine, Beni Suef University, Egypt
}

*Corresponding author: Safinaz Nagib Azab, Professor of Phoniatrics, Department of Otorhinolaryngology, Faculty of Medicine, Beni Suef University, Egypt

\begin{abstract}
Background: The use of cochlear implantation (CI) can fully restore hearing. Consequently, speech production can improve over time and enters the normal rang when traditional amplification Devices (hearing aids) are unable to restore access to the full range of phonemic components of speech, a cochlear implant (CI) is a widely used treatment option for children with sensorineural hearing loss (SNHL).

Purpose: The aim of this study is to compare the functional benefit of the communicative skills of children with CI without pre-implantation aural/oral rehabilitation in relation to those with CI with pre-implantation 6 months aural/oral rehabilitation in order to compare the role of pre-implantation aural/oral rehabilitation on the communicative abilities of severe to profound and profound sensorineural hearing impaired children.

Method: This study has a prospective design. It started after final diagnosis and decision that all children are candidates for CI but half of them are fitted with behind the ear hearing aids and the other half of children are immediately implanted provided that the primary language assessment before rehabilitation is present in the medical files of these children. A 2nd language assessment was done after 12 months of language therapy to detect the progress of the language development. These sixty patients were divided into two groups:
\end{abstract}

a)Group A: Thirty children, who have used behind the ear hearing aids for one year before CI and attended regular language therapy.

b) Group B: Thirty children, who shifted immediately to cochlear implantation, and were enrolled in auditory training and language therapy for one year.

Results: Total language age of children using cochlear implant without pre-implantation aural/oral rehabilitation is significantly higher than that in the children while using hearing aids for one year before CI. Also, there is highly significant difference between frontal and back speech sounds in the children after immediate implantation with positive correlation.

Conclusion: Cochlear implant is safe \& reliable technique. The fact that many profoundly hearing impaired children using immediate cochlear implant without pre-implantation aural/oral rehabilitation can develop functional levels of speech perception \& production, develop competency level in a language other than their primary language and continuation of language therapy together with proper mapping accordingly is a must to enroll these children in main stream education.

Keywords: Hearing Aids; Cochlear Implant; Language; Speech Intelligibility Pre-implantation Rehabilitation

Abbreviations: SNHL: Sensorineural Hearing Loss; HA: Hearing Aids; CI: Cochlear Implantation 


\section{Introduction}

Language in children begins to develop since birth and is nearly complete by the age of 6 years. Language skills, speech quality, expressive and receptive vocabulary are enhanced by exposure to aural language since as early an age as possible [1]. Children spend many hours in acoustic environments where target speech signals are embedded in competing sounds from multiple sources. In these environments, perception of target speech is assisted by a listener's a listener's ability to segregate the multitude of sounds into separate auditory streams, one cue to which is the angle of incidence of different sounds [2]. Children with profound sensorineural hearing loss (SNHL) experience delays in learning to understand the speech of others and to produce intelligible speech. There is solid evidence that moderate (or more severe) hearing impairment exerts a negative impact on speech, language, cognitive development, and early identification and management may be of great benefit to these children, through improved language, communication, mental health, and employment prospects [3]. The use of Hearing Aids (HA) or Cochlear Implantation (CI) can partially or fully restore hearing. Consequently, speech production can improve over time and enters the normal range. After hearing is restored, hearing impaired individuals use auditory feedback to adjust voice features such as voice intensity, intonation and vowel duration [4]. When traditional amplification devices (hearing aids) are unable to restore access to the full range of phonemic components of speech, a cochlear implant (CI) is a widely used treatment option for children with SNHL [5]. Cochlear Implants (CI) which are called as bionic ears are effective in trans- mitting salient features of speech, especially in quiet [6]. Because the goal of restored hearing in a deaf child is to enable useful hearing, a key measure of outcome should reflect how a deaf child's experience with a CI develops into the effective use of spoken language. Parental surveys indicate that the outcome of their greatest concern after surgical intervention in children with SNHL is the level of spoken language achieved [7]. Cochlear implants have become a popular option for children with profound hearing loss. Evidence supporting the benefits of early implantation is found in experimental [1], developmental [2], and clinical cochlear implant studies [3]. The consensus is that children have the best opportunity to learn language during their first 5 years of life. According to [2], this critical period for language learning is particularly important in deaf and hearing-impaired children. Providing cochlear implants to deaf children at a young age may enable them to take advantage of this critical period for learning language and is likely to increase their chances for developing speech and language skills like those of normal-hearing children. Early implantation would also result in a decrease in the duration of auditory deprivation, a decrease considered to positively influence performance with a cochlear implant [4].

\section{Objectives}

The aim of this study is to compare the functional benefit of the communicative skills of children with immediate CI without preimplantation aural/oral rehabilitation in relation to those using hearing aid with pre-implantation aural/oral rehabilitation in order to compare the role of each amplification device and the effect of pre-implantation aural/oral rehabilitation on the communicative abilities of severe to profound and profound sensorineural hearing impaired children.

\section{Subjects \& Methods}

This research was conducted during the period between the years 2017 and 2018. The study protocol was approved by the Otolaryngology Department Council of Beni-Suef University and Otolaryngology Department Council of King Abd Elaziz specialized hospital Jouf, Saudi Arabia. Consent to participate in this research was obtained from the subjects' parents before commencement of the study. This study employed a comprehensive design to examine outcomes in multiple domains of communication in children who used either bilateral behind the ear hearing aids and preimplantation aural/oral rehabilitation or an immediate unilateral cochlear implant without pre-implantation rehabilitation for a period of one year. These were selected from children seeking language rehabilitation in Phoniatrics Unit, Beni-Suef University Hospital and children seeking language rehabilitation in Phoniatrics Clinic, King Abd Elaziz specialized hospital Jouf, Saudi Arabia. Shortly after confirmation of bilateral permanent hearing loss, thirty children were typically fitted with bilateral behind the ear hearing aids using the desired sensation level (DSL) prescription method and regularly attend aural/oral rehabilitation sessions. Thirty children underwent a comprehensive team evaluation for cochlear implant candidacy and received immediate unilateral cochlear implants without pre-implant aural/oral rehabilitation. All children received audiologic management and preschool rehabilitation and all children were enrolled in rehabilitation programs with a focus on the development of receptive \&expressive language. Children were regular in Phoniatrics clinic, were asked to follow up auditory rehabilitation \& language therapy program twice per week. Children with cochlear implants were followed every month for mapping of their speech processor and speech recognition testing. The study received ethical approval from the Hospital of Beni-Suef University and from King Abd Elaziz specialized hospital and written informed consent was obtained from all their parents. Collaboration between ENT clinic, Audiology clinic\& Phoniatrics clinic was done in the form of ENT examination, audiological assessment, and language assessment and rehabilitation for all children. This study has a prospective design. It started after fitting half of the children with bilateral behind the ear hearing aids with aural/oral rehabilitation sessions and the other half of the children are immediately implanted, mapped, and regularly attended aural rehabilitation sessions provided that the primary language assessment before rehabilitate- ton is present in the medical files of all children. A 2nd language assessment was done after 12 months of language therapy to detect the progress of the language and the efficacy of pre-implantation aural/oral rehabilitation. These sixty patients were divided into two groups:

a) Group A: Thirty children, who have used behind the ear hearing aids for one year and attended regular language therapy despite those children, are candidates for cochlear implants. 
b) Group B: Thirty children, who shifted immediately to cochlear implantation and had regular language therapy and were enrolled in auditory training.

Half patients were fitted with bilateral powerful digital signal processing BEHAs and used them for at least a 12-months period before CI. Hearing aid use was determined by parental and therapist reports. After surgical implantation of the device and an adequate healing period for the other half of the patients, the implants were activated (usually 4 weeks after surgery). The children were fitted with one of the two brands of speech processors using a behind the ear controller. Speech processors used in this study were OPUS 2 with standard Sonata electrode \& Cochlear Freedom Processor with nucleus $24 \mathrm{k}$ straight electrode.

In this study using Modified Preschool Language Scale \& Subjective Speech Intelligibility Test gave us a summary of the improvement of these children. This is matched with other studies which focused that both comprehension and expression of spoken language are important markers of parent-perceived success of a CI (Figure 1).

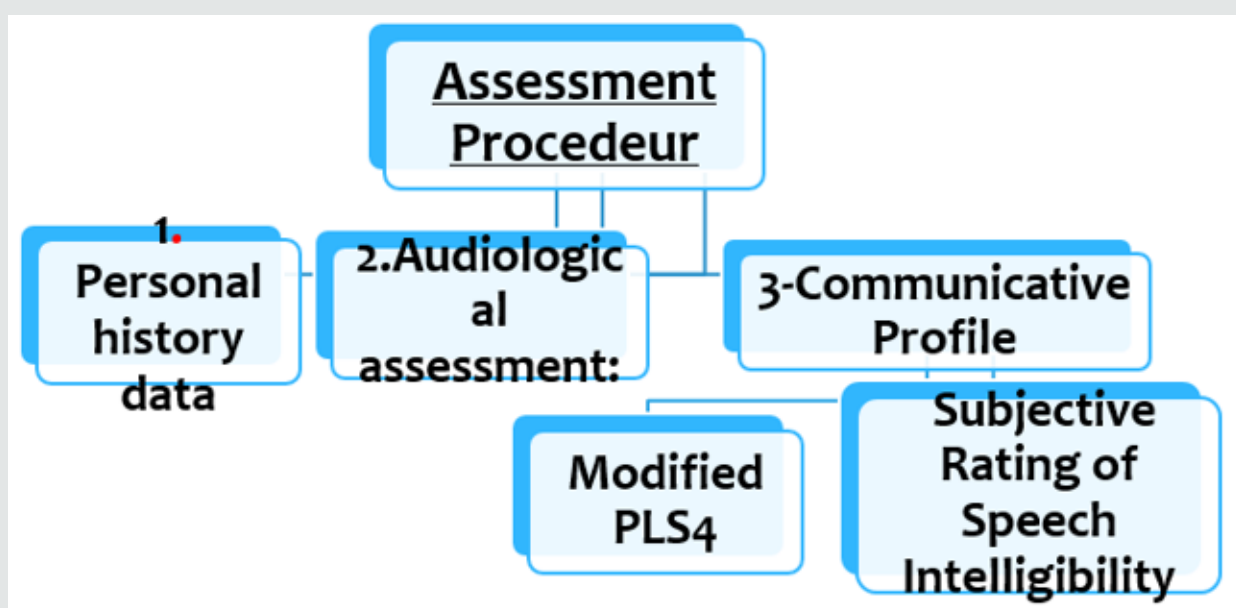

Figure 1.

Language Improvement Quotient: The language improvement quotient [8] was used to compare between the rates of progress in language in order to overcome the bias of age matching between the individuals in the study.

Language Improvement $=$ 2nd language age -1 st language age divided by duration of language rehabilitation.

a) A1 refers to language development of group (A) after using bilateral behind the ear hearing aids for 12 months which is calculated by this equation:

$\mathrm{A} 1=2$ nd language age -1 st language age divided by 12 (duration of rehabilitation)

b) B1 refers to language development of group (B) after using unilateral CI for 12 months which is calculated by this equation:

B1= 2nd language age -1st language age divided by 12 (duration of rehabilitation).

Speech analysis was performed using the Ain Shams assessment protocol which includes analysis of supra-segmental phonology (rate, stress and tonality), segmental phonology (consonants and vowels), nasal resonance and general intelligibility of speech. Assessment of auditory perception skills was performed evaluating a hierarchy of listening skills ranging from detection, to discrimination, identification, recognition and comprehension. Assessment of speech reading abilities was done and expressed as percent change over time.

\section{Statistical Studies}

Data was analyzed using SPSS, Statistical Package for the Social Sciences version 17 (SPSS Inc., Chicago, IL). Numerical data were expressed as mean, standard deviation, and range. For quantitative data, comparison was done using Mann-Whitney test (non-parametric t-test). A p-value $<0.05$ was considered significant. Spearman-rho method was used to test correlation between numerical variables $(r>0.3=$ no correlation, $r=0.3-0.5=$ fair correlation, $r=0.5-0.1$ = good correlation).

\section{Results}

Group (A) are hearing aids users for one year, Group (B) are CI users for 1 year. Demographic data of the 2 groups:
a) Age
b) Gender.
c) Incidence of hearing loss.
d) Psychometric evaluation.
e) Pure tone Audiometry.
f) First language age.
g) Radiology.

a) Age Distribution: Both groups are matched according to age. In group (A) the age of the children ranged between 3 years and 7years. In group (B). The age of the children ranged 
Between 3 years\& 7 years, provided that all children were implanted before the age of 6 years.

b) Gender: No significant difference was noted in gender of both groups.

c) Incidence of Hearing Loss: In group (A) there were 24 children $(80 \%)$ with congenital hearing impairment and 6 children $(20 \%)$ with acquired hearing loss, while in group (B) there were 18 children (60\%) with congenital hearing impairment and 12 children (40\%) With acquired hearing loss.

d) Psychometric Evaluation: All children in group (A) had normal psychometric evaluation with a mean Value 87.5 \pm 4.6; also, in group (B) all children had normal psychometric Evaluation with a mean value 86.4 \pm 5.1 .

e) Pure Tone Results: Pure tone results of group (A) maintained a mean value of 27.9 dB HL. Group (B). Decreased in mean values from $65.7 \pm 8.2 \mathrm{~dB}$ HL. There was a highly significant difference $(\mathrm{P}=0.001)$ between group $(\mathrm{A})$ and group (B) in favor of group (B).

f) First Language Age: Before start of therapy, both groups had no passive vocabulary and were Nonverbal. They used either babbling or vocal play.

g) Radiology: All Children both in groups (A) and (B) were having normal CT and MRI of Petrous bone.

Tables 1-3 demonstrate the progress of the language abilities, the auditory abilities and the speeding reading abilities of both groups, respectively, from the time just prior to the rehabilitation (either oral \aural in group A or aural in group B) as compared to the evaluation done one year the rehabilitation (Table 4).

Table 1: Results of collective language improvement in both groups using paired-T test.

\begin{tabular}{|c|c|c|c|c|}
\hline & $\begin{array}{c}\text { Language age before 1 } \\
\text { year rehabilitation }\end{array}$ & $\begin{array}{c}\text { Language age after 1 year } \\
\text { rehabilitation }\end{array}$ & Paired-T test & Significance \\
\hline Group A & 1 year5m \pm 1 year8m & $1 \mathrm{y} 6 \mathrm{~m} \pm 1 \mathrm{y} 3 \mathrm{~m}$ & 0.2 & $\mathrm{P}>0.05 \mathrm{NS}$ \\
\hline Group B & $1 \mathrm{y} 10 \mathrm{~m} \pm 7 \mathrm{~m}$ & $5 \mathrm{y} 2 \mathrm{~m} \pm 1 \mathrm{y} 7 \mathrm{~m}$ & 6.2 & $\mathrm{P}<0.01 \mathrm{HS}$ \\
\hline
\end{tabular}

Table 2: Results of improvement in the auditory abilities of the 2 groups after 1 year rehabilitation using paired-T test.

\begin{tabular}{|c|c|c|c|c|}
\hline & Before rehabilitation & After 1 year rehabilitation & Paired-T test & Significance \\
\hline Group A & $5 \% \pm 6 \%$ & $81 \% \pm 17 \%$ & 16.5 & $\mathrm{P}>0.05 \mathrm{NS}$ \\
\hline Group B & $2 \% \pm 4 \%$ & $56 \% \pm 21 \%$ & 7.9 & $\mathrm{P}<0.01 \mathrm{HS}$ \\
\hline
\end{tabular}

Table 3: Results of progress in speech reading ability before and after rehabilitation in the 2 groups (using Paired-T test).

\begin{tabular}{|c|c|c|c|c|}
\hline & Before rehabilitation & After 1 year rehabilitation & Paired-T test & Significance \\
\hline Group A & $100 \% \pm 0$ & $19 \% \pm 13 \%$ & 6.6 & P $>0.05$ NS \\
\hline Group B & $100 \% \pm 0$ & $58 \% \pm 21 \%$ & 7.9 & P $<0.01 \mathrm{HS}$ \\
\hline
\end{tabular}

Table 4: Difference in the speech ratings between the 2 groups after 3 years of rehabilitation (using Mann-Whitney test).

\begin{tabular}{|c|c|c|c|c|}
\hline Speech Skills & Group A & Group B & Z - Value & Significance \\
\hline Rate & $0.6 \pm 0.5$ & $1.6 \pm 0.7$ & 2.7 & $\mathrm{P}<0.01 \mathrm{HS}$ \\
\hline Stress & $0.6 \pm 0.4$ & $2.0 \pm 0.5$ & 3.8 & $\mathrm{P}<0.01 \mathrm{HS}$ \\
\hline Tonality & $0.4 \pm 0.5$ & $1.7 \pm 1.0$ & 2.7 & $\mathrm{P}<0.01 \mathrm{HS}$ \\
\hline Consonants & $1.0 \pm 0$ & $2.1 \pm 0.6$ & 3.7 & $\mathrm{P}<0.01 \mathrm{HS}$ \\
\hline Vowels & $0.9 \pm 0.3$ & $2.0 \pm 0.7$ & 3.3 & $\mathrm{P}<0.01 \mathrm{HS}$ \\
\hline Resonance & 0 & $1.0 \pm 0.9$ & 3.9 & $\mathrm{P}<0.01 \mathrm{HS}$ \\
\hline General intelligibility & $0.8 \pm 0.4$ & $2.2 \pm 0.4$ & 3.3 & $\mathrm{P}<0.01 \mathrm{HS}$ \\
\hline Voice & $0.8 \pm 0.6$ & $2.0 \pm 0.5$ & & \\
\hline
\end{tabular}

\section{Discussion}

The primary purpose of this study was to obtain comprehensive data on the development of language and speech skills in a group of permanent hearing impaired children. This group shared the common degree of bilateral hearing impairment (severe to profound or profound hearing impairment), they all sought amplification, and they all sought language rehabilitation after receiving amplification using primarily auditory-based cues. The study aimed also to investigate the difference between the language and speech development under two amplification conditions; bilateral behind the ear hearing aids and unilateral cochlear implants. The choice of language age deficit to compare language skills development among the studied groups is justified by the fact that three variables usually co-vary when language results are analyzed in children; age of use of the amplification device whether hearing aid (HAs) or cochlear implant (CI), the language age before start of rehabilitation, and the language age of children after the time of rehabilitation. The difference in ages at evaluation places the younger children at a maturational and developmental disadvantage in comparison with their older peers. Thus, analyzing the results in terms of language 
age scores might put the younger group at a disadvantage. At the same time, analyzing the results in terms of language age deficits, although more reasonable, but still, in theory, puts the older group at a disadvantage because of the impact of their ages giving higher values for the deficit from the scored language age. That's why the hypotheses of using the language improvement quotient [8] after determining the exact language age, may be more realistic and less biased by the chronological age differences at the time of evaluation. In this study using Modified Preschool Language Scale \& Subjective Speech Intelligibility Test gave us a summary of the improvement of these children. A perfect model for comparing the results of both devices may be practically impossible, given the current indications of cochlear implant use. In this study, which was applied on two groups with comparable ages, a comparison was made between the outcomes of the 2 devices along a period of (re)habilitation of one year in their course of therapy. If the CI group were doing better than the HA group, it would indicate that the selection criteria were too conservative and some of the HA users might be better off with a CI. This raises the suspicion of the fact that HA users plateau after a period of little progress or at least their progress continue at a less pace. Cochlear implants may have a superior effect on the acoustic environment of children more than hearing aids. During the 90s of the last centuries, and using the early models of speech processes, studies proved that CI users gained better results than HA users in language and perception skills [9-14]. The minimum age for implantation has progressively reduced [15]. Advantages of cochlear implants over hearing aids extended also the adult population [15]. In a study by [16], they found CIs and children with HAs, aged 4 to 5 years, differ significantly on language abilities and there were differences in articulation skills in favor of the CI users. Advances in sound processors and related software have enhanced the fidelity with which complex sounds are processed into physiologically meaningful codes [17]. This study pointed to the importance of conducting comprehensive assessments when evaluating whether a child with severe to profound sensory neural hearing loss would likely derive greater benefit from a cochlear implant compared to a hearing aid. To date, only a few systematic studies have involved large numbers of children who received implants at various ages and have investigated both the effects of age at implantation and the amount of experience with an implant. Most of such studies were concerned with the speech perception skills after cochlear implantation with a clear evidence of the effect of early implantation on rate of acquisition of such perception skills when they are implanted at $2-4$ years of age [18]. Concerning the auditory abilities, the progress imposed by the effect of cochlear implantation group produced better abilities than the hearing aids group in the auditory abilities. This may be explained by the fact that the hearing aids group were more rigid to their habits of relying on their visual cues making the children less efficient in acquiring the training proficiency provided to them during therapy sessions. In an explanation of this, [19] described recruitment of the auditory cortex by the visual and somatosensory systems in congenitally deaf humans. They reported that the extent of crossmodal recruitment of the auditory cortex increases as the duration of deafness increases, deterring the restoration of auditory processing in the auditory cortex of long-term deafened individuals after cochlear implantation. They also suggested that the age beyond which the effects of cross-modal plasticity in the auditory cortex are more difficult to reverse is about 6.5 years. It has also been documented that there is a change in the cochlear place code during development [20]. This may be necessary for the formation of normal and effective connections between auditory centers and for the proper development of elements within the central auditory pathways. Early cochlear implantation may contribute to the maintenance of these important developmental milestones.

\section{Conclusion}

CI children showed better rate of language acquisition skills along a one-year use of the implant compared to a similar period of HA group of HA users. The implanted group demonstrated significantly better auditory abilities, better speech production skills, and better speech intelligibility one year after implantation and with aural rehabilitation - than the aided group with oral \aural rehabilitation. The implanted group also ended with significantly less or no speech reading abilities than the aided group one year after implantation. These results indicate the favorable effect implantation over the previous parameters. Language skills shows a significant difference between the two groups. Consequently, oral \aural rehabilitation with hearing aids - even for few monthsis not mandatory.

\section{References}

1. Holt RF, Shirks MA (2008) An exploratory look at pediatric cochlear implantation: is earliest always best? Ear Hear 29: 492-511.

2. Mahmoudi Z, Rahati S, Ghasemi M, Asadpour V, Tayarani H, et al. (2011) Classification of voice disorders in children with cochlear implantation and hearing aid using multiple classifier fusion. Biomed Eng. Online 10: 3

3. Kennedy C, McCann D, Campbell MJ, Kimm L, Thornton R (2005) Universal newborn screening for permanent childhood hearing impairment: an8-year follow-up of a controlled trial. Lancet 366(9468): 660- 662.

4. Amirfattahi R, Sheikhzade H (2001) Analyzing alterations in vowel articulation after using multichannel cochlear implant in children without hearing antecedent. Journal of Amirkabir polytechnic University 46: 209- 227 .

5. Kral A, Eggermont JJ (2007) What's to lose and what's to learn development under auditory deprivation, cochlear implants and limits of cortical plasticity. Brain Res Rev 56: 259-269.

6. Kate Gfeller, Dingfeng Jiang, Jacob Oleson, Virginia Driscoll, Carol Olszewski, et al. (2012) The Effects of Musical andLinguistic Components in Recognition of Real-World Musical Excerpts by Cochlear Implant Recipients and Normal-Hearing Adults. J Music Ther 49: 68-101.

7. Nikolopoulos TP, Dyar D, ODonoghue GM, Archbold S (2004) Development of spoken language grammar following cochlear implantation in prelingually deaf children. Arch Otolaryngology Head Neck Surg 130: 629-633.

8. Bassiouny S, Hegazi M, Saber A, S Hassan (2006) The effect of age at implantation on the language and speech of cochlear implanted children: A 3-years follow-up. Ain Shams Medical Journal 57: 1307-1321.

9. Moog JS, Geers AE (2003) Epilogue: major findings, conclusions and implications for deaf education. Ear Hear 24:121-125.

10. Anderson I, Weichbold V, D Haese PS, Szuchnik J, Quevedo MS, et al. (2004) Cochlear Implantation in children under the age of two - what do the outcomes show us? Int J Pediatr Otorhinolaryngol 68:425-431. 
11. Calmels MN, Saliba I, Wanna G, Cochard N, Fillaux J, et al. (2004) Speech perception and speech intelligibility in children after cochlear implantation. Int J Pediatric Otorhinolaryngology 68: 347-351.

12. Manrique M, Cervera Paz FJ, Huarte A, Molina M (2004) Advantages of cochlear implantation in prelingual deaf children before2 years of age when compared with later implantation. Laryngoscope 114: 1462-1469.

13. Hocevar Boltezar I, Vatovec J, Gros A, Zargi M (2005) The influence of cochlear implantation on some voice parameters. Int J Pediatr Otorhinolaryngol 69: 1635-1640.

14. Geers AE, Moog JS, Biedenstein J, Brenner C, Hayes H (2009) Spoken language scores of children using cochlear implants compared to hearing age-mates at school entry. J Deaf Stud Deaf Educ 14: 371- 385.

15. Vincenti V, Bacciu A, Guida M, Marra F, Bertoldi B, et al. (2014) Pediatric cochlear implantation: an update. Ital J Pediatric 40: 72.
16. Fitzpatrick EM, Crawford L, Ni A, Durieux Smith A (2011) A descriptive analysis of language and speech skills in 4- to 5-yr-old children with hearing loss. Ear Hear 32: 605-616.

17. Timothy MMarkman, Alexandra LQuittner, Laurie SEisenberg, Emily ATobey, Donna Thal, et al. (2011) Language development after cochlear implantation: an epigenetic model J Neurodev Disord 3: 388-404.

18. Cheng AK, Grant GD, Niparko JK (1999) Meta-analysis of pediatric cochlear implant literature. Ann Otol Rhinol Laryngol 177: 124-128.

19. Lee DS, Lee JS, Oh SH, Kim SK, Kim JW, et al. (2001) Cross-modal plasticity and cochlear implants. Nature 409: 149-150.

20. Ryals BM, Rubel EW, Lippe W (1991) Issues in neural plasticity as related to cochlear implants in children. Am J Otol 12 (suppl): 22-27.

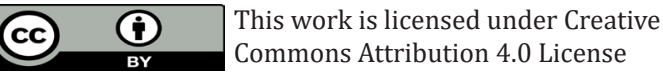

To Submit Your Article Click Here:

Submit Article
DOI: $10.32474 /$ SJO.2019.02.000129

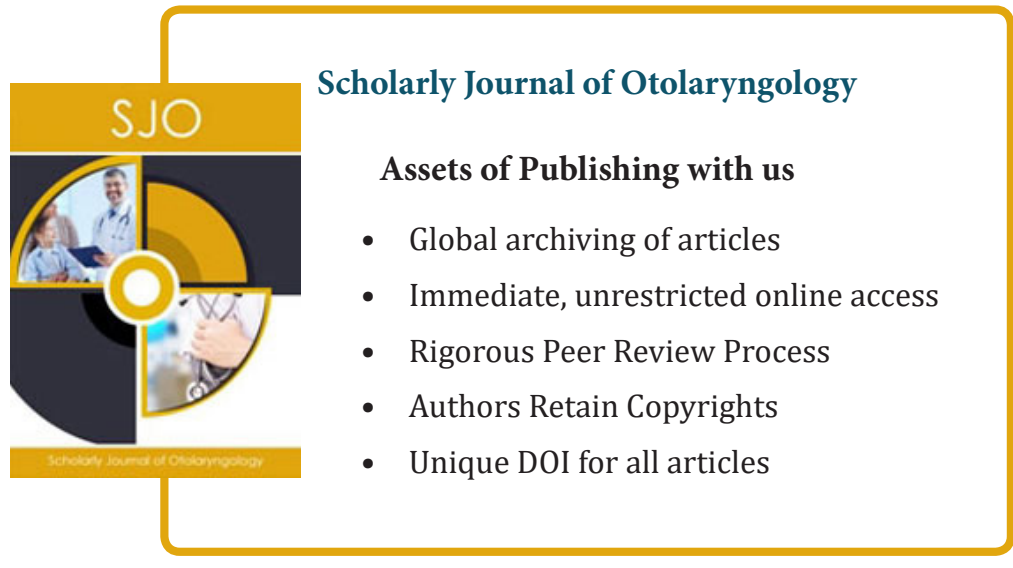

